E3S Web of Conferences 1, 15012 (2013)

DOI: $10.1051 / \mathrm{e} 3$ sconf/20130115012

(c) Owned by the authors, published by EDP Sciences, 2013

\title{
lodine Contents in Baby Food Consumed in Japan
}

\author{
M. Yoshida $^{1}$, S. Nozaki ${ }^{1}$ and K. Inui ${ }^{1}$ \\ ${ }^{1}$ Laboratory of Food and Nutritional Sciences, Faculty of Chemistry, Materials and Bioengineering, Kansai University, \\ Suita, Osaka 564-8680, Japan.hanmyou4@kansai-u.ac.jp
}

\begin{abstract}
To evaluate iodine intake in Japanese infants, iodine contents were determined in both commercial and homemade baby food samples consumed in Japan. Fifty-three samples of commercial bottled or retort baby food and 25 samples of homemade baby food for one day were collected and their iodine contents were determined by inductively coupled plasma mass spectrometry after an extraction with $0.5 \%$ tetramethylammonium hydroxide. Among the commercial baby food samples, 35 samples showed low iodine values $(<50 \mathrm{ng} / \mathrm{g}$ wet weight), while 8 samples showed markedly high iodine values $(>1000 \mathrm{ng} / \mathrm{g}$ wet weight). Significantly higher iodine values were observed in 15 samples composed of dishes cooked using kombu (a kind of kelp) than other samples. Among the homemade baby food samples, 12 samples brought very low iodine intake $(<1-24 \mu \mathrm{g} / \mathrm{d})$, while 5 samples brought very high iodine intake (283-978 $\mu \mathrm{g} / \mathrm{d})$. These results indicate that intermittent high iodine baby food including dishes cooked using kombu contributes to sufficient iodine intake in Japanese infants.
\end{abstract}

Key words: Iodine, baby foods, inductively coupled plasma mass spectrometry, kombu (a kind of kelp)

\section{Introduction}

Iodine is an essential component of thyroid hormone. Seaweeds contain iodine at a very high level; in particular, kombu (several kinds of Saccharina $s p$, a kind of kelp) contains it at a level of more than $2 \mathrm{mg} / \mathrm{g}$. Kombu is a traditional Japanese foodstuff and many Japanese eat it routinenly. As a result, the Japanese average iodine intake is over the tolerable upper intake level of iodine for the US and European people (Zava and Zava, 2011). In a general Japanese, there is almost no report of the health disturbance by excess iodine intake. However, in the Japanese infants, a hypothyroidism by excess iodine intake has been reported (Nishiyama et al. 2004) In the present study, to evaluate iodine intake in Japanese infants, iodine contents were determined in both commercial and homemade baby food samples consumed in Japan

\section{Materials and Methods}

Fifty-three samples of commercial bottled or retort baby food were purchased from 4 Japanese food companies. Among the samples, 24 samples were for $\geq 7$-month-old baby and 29 samples were for $\geq 9$-month-old baby. Twenty-five samples of homemade baby food for one day were collected from Japanese mothers in child-rearing.

The baby food sample was freeze-dried, homogenized and milled. Iodine in the dried samples was extracted with $0.5 \%$ tetramethylammonium hydroxide (TMAH). Two hundred milligrams of the dried samples was mixed with $40 \mathrm{~mL}$ of $0.5 \%$ TMAH and left overnight. The mixture was heated at $60^{\circ} \mathrm{C}$ for 6 $\mathrm{h}$ and centrifuged. The supernatant was filtrated through a 0.45 $\mu \mathrm{m}$ membrane filter. Iodine were determined by inductively coupled plasma mass spectrometry using tellurium as an internal standards.

\section{Results and Discussion}

Fig. 1 shows iodine contents in 53 commercial baby food samples. Among the samples, 35 samples showed low iodine values $(<50 \mathrm{ng} / \mathrm{g}$ wet weight), while 8 samples showed markedly high iodine values $(>1000 \mathrm{ng} / \mathrm{g}$ wet weight). Among the commercial baby food samples, 15 samples were composed of dishes cooked using kombu. Fig. 2 shows effect of kombu use on the iodine contents. Significantly higher iodine values were observed in these 15 samples than other samples. Among the samples without kombu, 3 samples showed high iodine values. These 3 samples were composed of dishes cooked using hijiki (Sargassum fusiforme, a kind of edible sea weed).

Fig. 3 shows estimated daily iodine intake from homemade baby food. Among the homemade baby food samples, 13 samples brought very low iodine intake $(<25 \mu \mathrm{g} / \mathrm{d})$, while 9 samples brought very high iodine intake $(>250 \mu \mathrm{g} / \mathrm{d})$. These results indicate that intermittent high iodine baby food including dishes cooked using kombu contributes to sufficient iodine intake in Japanese infants. 


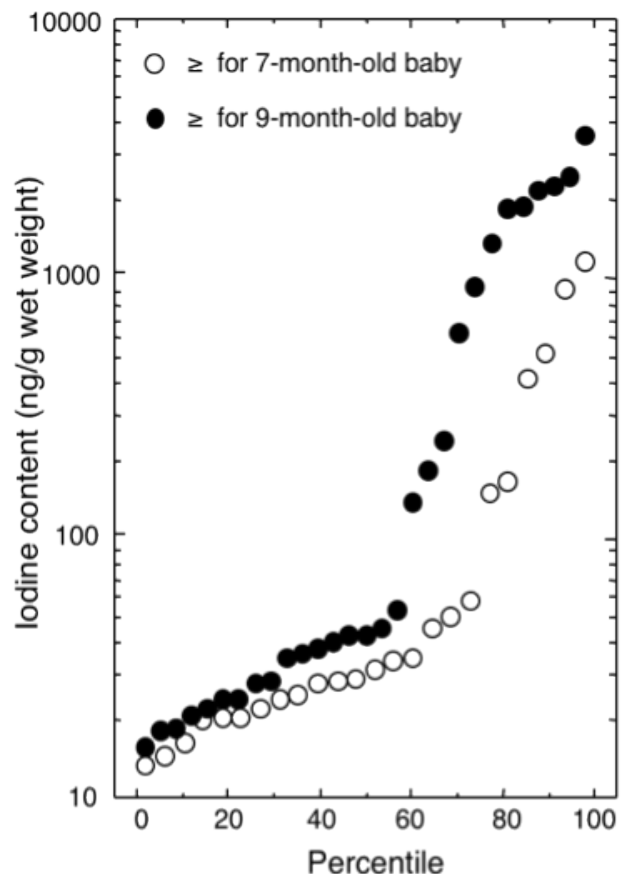

Fig. 1 Percentile curve of iodine contents in 53 commercial baby food samples.

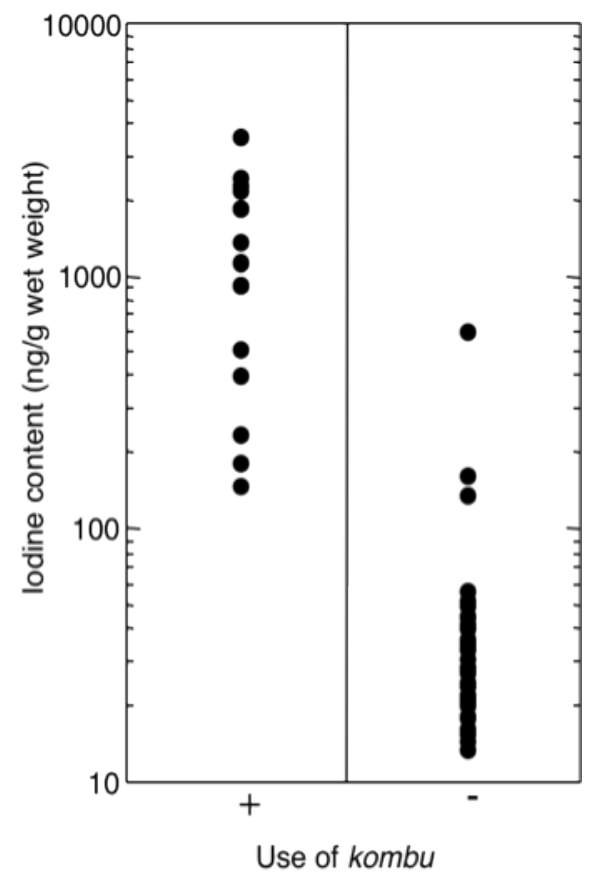

Fig. 2 Effect of kombu use on iodine content in baby food.

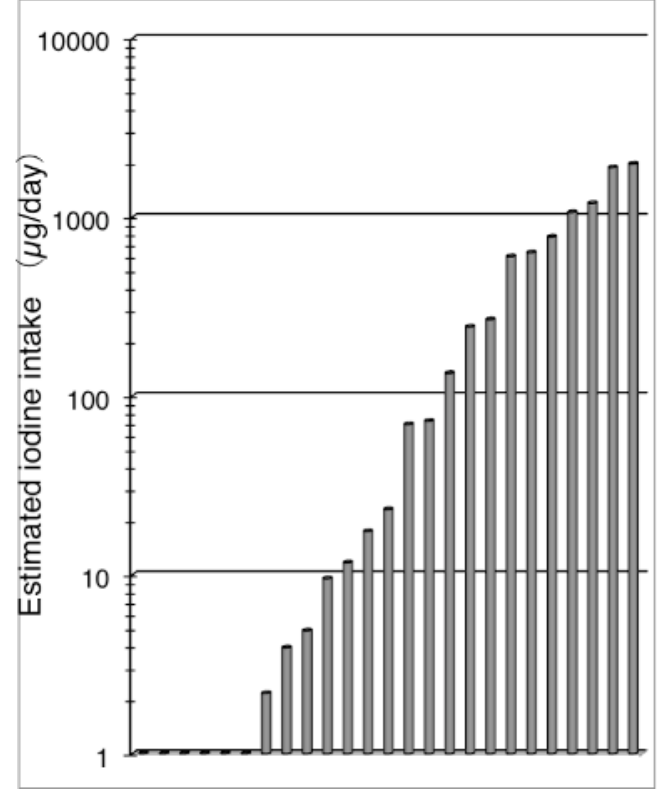

Fig.3 Estimated daily iodine intake from 25 samples of homemade baby food

\section{Acknowledgments}

This study was supported by a grant for comprehensive research on cardiovascular and lifestyle disease from the Ministry of Health, Labour and Welfare of Japan.

\section{References}

Nishiyama S, Mikeda T, Okada T, Nakamura K, Kotani T, Hishinuma A. Transient hypothyroidism or persistent hyperthyrotropinemia in neonates born to mothers with excessive iodine intake. Thyroid. 2004; 14:1077-83.

Zava TT, Zava DT. Assessment of Japanese iodine intake based on seaweed consumption in Japan: A literature-based analysis. Thyroid Res. 2011; 4:14. 